\title{
Study of Dielectric Properties and Ultrasonic Attenuation in KDP-Type Ferroelectrics
}

\author{
Vijay Singh Bist and Narayan Singh Panwar \\ USIC, HNB Garhwal University, Srinagar, Garhwal, Uttrakhand 24 6174, India \\ Correspondence should be addressed to Vijay Singh Bist; vsbist_usic68@yahoo.com
}

Received 12 October 2015; Accepted 19 January 2016

Academic Editor: Sergey B. Mirov

Copyright ( 2016 V. S. Bist and N. S. Panwar. This is an open access article distributed under the Creative Commons Attribution License, which permits unrestricted use, distribution, and reproduction in any medium, provided the original work is properly cited.

\begin{abstract}
The soft mode dynamical model has been used to study dielectric properties and ultrasonic attenuation in KDP-type ferroelectric crystals. The model Hamiltonian proposed by Blinc and Zeks has been modified by considering lattice anharmonicity up to fourthorder. The correlations appearing in the dynamical equation have been evaluated using double-time thermal retarded Green's functions method and Dyson's equation. Without any decoupling, the higher order correlations, appearing in the dynamical equation, have been evaluated using the renormalized Hamiltonian. The expressions for collective frequencies, width, dielectric constant, ultrasonic attenuation, and tangent loss have been calculated. The dielectric properties and ultrasonic attenuation strongly depend on the relaxational mode behavior of stochastic motion of $\mathrm{H}_{2} \mathrm{PO}_{4}$ group in KDP-type ferroelectrics. By fitting model values of physical quantities, the temperature dependence of $\left\langle S_{q}^{z}\right\rangle$ and $\left\langle S_{q}^{x}\right\rangle$ for different value of four-body coupling coefficient and dielectric constant and loss tangent has been calculated. The calculated and observed results have been found in good agreement.
\end{abstract}

\section{Introduction}

The oscillations of atoms in solids are responsible for different characteristics, such as specific heat, optical, dielectric, and electrical properties. The anharmonicity in solids is responsible for existence of thermal expansion, temperature variation of elastic constants, lattice thermal conductivity, deviation of specific heat from Dulong-Petit law at high temperature, existence of ferroelectricity in certain materials, and so forth. Many attempts have been made theoretically and experimentally to find the explanation of these phenomena in terms of anharmonicity. Extensive reviews [1,2] are available discussing the contribution of anharmonicity to various properties of crystals.

In order-disorder type ferroelectrics, as Potassium Dihydrogen Phosphate $\left(\mathrm{KH}_{2} \mathrm{PO}_{4}\right)$, the transition is associated with the tunneling of proton through a barrier between two positions of minimum potential energy in double well potential in the hydrogen bond at the transition temperature [3]. Busch [4] was the first to show that $\mathrm{KDP}\left(\mathrm{KH}_{2} \mathrm{PO}_{4}\right)$ exhibits a phase transition at low temperature. $\mathrm{KDP}$ is prototype of a family of crystals with bridging hydrogen bonds and its physical properties have been extensively studied [3, 5-10].

Kaminow and Damen [11] first observed the soft mode associated with the ferroelectric phase transition of the KDPtype crystal at $122.3 \mathrm{~K}$ by measuring the low frequency Raman scattering in $x(y x) y$ configuration. Since then $B_{2}(z)$ soft mode which is connected to the susceptibility along the crystalline $c$-axis through the Lyddane-Sachs-Teller relation [12] has been extensively studied by Scott [13] and interpreted using the pseudospin model [14-16] and its modifications in $[3,17]$. In these theories, such a particular mode of proton motions along hydrogen bonds in $a-b$ plane is coupled to other ion modes, bearing an electrical dipole moment along $c$-axis is considered to play an essential role for the ferroelectric transition, and therefore little attention has been paid to the modes other than $B_{2}(z)$ soft mode. In KDP crystal, however, there are four tunneling protons in a primitive unit cell of the paraelectric (hereafter referred to as PE) phase and consequently four normal modes are belonging to $B_{2}(z)$, a doubly degenerate $E(x, y)$, and $A_{2}$ modes [18]. $E(x, y)$ mode, which is both infrared and Raman active, provides valuable information, because this mode and $B_{2}(z)$ mode 
reflect the nature of collective proton motion which triggers the phase transition, and, moreover, in contrast to $B_{2}(z)$, it is directly related to the polarization along the crystalline $a$ axis.

At first, Pak [19] employed Green's function methods in the order-disorder type ferroelectrics and, however, did not consider the anharmonic interactions. The phonon anharmonic interactions have been found very important in explaining dielectric, thermal, and scattering properties of solids by many authors $[6-9,20,21]$ in the past. Pak's theory was further developed by Ramakrishnan and Tanaka [22], who calculated the excitation spectrum of the system but did not consider the anharmonic interactions. Their attempt, however, established the superiority of Green's function method over the other methods. Ganguli et al. [23] modified Ramakrishnan and Tanaka theory by considering anharmonic interaction. Their treatment explains many features of order-disorder ferroelectrics. However, due to insufficient treatment of anharmonic interactions, they could not explain quantitatively good results and could not describe some very interesting properties, like dielectric properties, ultrasonic attenuation, relaxation rate, and so forth.

In the present study, the four-particle cluster model Hamiltonian with the phonon anharmonicity up to fourthorder has been taken to theoretical study of dielectric properties and ultrasonic attenuation in KDP-type crystals, using double time Green's functions method and Dyson's equation. Proton Green's function and phonon Green's function have been solved for the collective system. Expressions for collective mode frequency shifts, widths, transition (Curie) temperature, and the expectation value of the proton collective mode components at site $q\left(\left\langle S_{q}^{z}\right\rangle,\left\langle S_{q}^{x}\right\rangle\right)$ have been derived and discussed in KDP-type crystals. By fitting model values of physical quantities, the temperature dependence of $\left\langle S_{q}^{z}\right\rangle$ and $\left\langle S_{q}^{x}\right\rangle$ for different value of four-body coupling, dielectric constant, and loss tangent has been calculated. The calculated and observed results have been found in good agreement.

\section{The Model Hamiltonian and Green's Function}

For KDP crystal, the four-particle cluster model Hamiltonian [24] along with third- and fourth-order phonon anharmonic interaction terms is expressed as

$$
\begin{aligned}
H= & -2 \Omega \sum_{i} S_{i}^{x}-\frac{1}{2} \sum_{i j} J_{i j} S_{i}^{z} S_{j}^{z}-\frac{1}{4} \sum_{i j k l} J_{i j k l}^{\prime} S_{i}^{z} S_{j}^{z} S_{k}^{z} S_{l}^{z} \\
& +\frac{1}{4} \omega_{k}\left(A_{k}^{+} A_{k}+B_{k}^{+} B_{k}\right)-\sum_{i} \bar{V}_{i k} S_{i}^{x} A_{k} \\
& +\sum_{\vec{k}_{1}, \vec{k}_{2}, \vec{k}_{3}} V_{3}\left(\vec{k}_{1} \vec{k}_{2} \vec{k}_{3}\right) A_{\vec{k}_{1}}, A_{\vec{k}_{2}}, A_{\vec{k}_{3}} \\
& +\sum_{\vec{k}_{1}, \vec{k}_{2}, \vec{k}_{3}, \vec{k}_{4}} V_{4}\left(\vec{k}_{1} \vec{k}_{2} \vec{k}_{3} \vec{k}_{4}\right) A_{\vec{k}_{1}}, A_{\vec{k}_{2}}, A_{\vec{k}_{3}}, A_{\vec{k}_{4}},
\end{aligned}
$$

where the first two terms constitute the original pseudospin model Hamiltonian and the third is the quadrupole contribution (the four-body interaction). $S_{i}^{x}$ is the tunneling operator which measures the tunneling power of the proton between the hydrogen double well, $\Omega$ the tunneling frequency, and $S_{i}^{z}$ the half of the difference of the occupation probabilities for the proton in the two equilibrium positions of hydrogen bond. $J_{i j}$ is the two-body coupling coefficient and is the same for every pair of protons in KDP and the fourbody coupling coefficient, and $J_{i j k l}^{\prime}$ refers to the four hydrogen bonds in the $\mathrm{PO}_{4}$ group in KDP. In the last fourth terms $\omega_{k}$ is bare phonon frequency, $A_{k}$ and $B_{k}$ are displacement and momentum operators, $\bar{V}_{i k}$ is proton-lattice interaction term, and $V_{3}$ and $V_{4}$ are the third- and fourth-order anharmonic coefficients.

2.1. Collective Proton Wave Width and Shift. The correlations appearing in the proton response function can be evaluated using double time thermal retarded Green's function [25] using the symmetrical decoupling scheme, after applying Dyson's treatment:

$$
\begin{aligned}
G_{q q^{\prime}}^{z z}\left(t-t^{\prime}\right) & =\left\langle S_{q}^{z}(t) ; S_{q^{\prime}}^{z}\left(t^{\prime}\right) 》\right. \\
& =-j \theta\left(t-t^{\prime}\right)\left\langle\left[S_{q}^{z}(t), S_{q^{\prime}}^{z}\left(t^{\prime}\right)\right]\right\rangle,
\end{aligned}
$$

where the angular brackets denote the average over the large canonical ensemble and $\theta(t)$ is the Heaviside step function having properties

$$
\begin{array}{ll}
\theta(t)=1 & \text { for } t>1, \\
\theta(t)=0 & \text { for } t<1, \quad j=(-1)^{1 / 2} .
\end{array}
$$

Differentiating (2) twice with respect to time " $t$," using Hamiltonian (1) taking Fourier transformation, one obtains

$$
\left(\omega^{2}-\Omega^{2}\right) G_{q q^{\prime}}^{z z}=\frac{\Omega\left\langle S_{q}^{x}\right\rangle \delta_{q q^{\prime}}}{\pi}+\frac{\Omega}{\pi} E(\omega),
$$

where $E(\omega)$ is higher order Green's functions:

$$
E(\omega)=\left\langle\left\langle F_{q}(t) ; S_{q^{\prime}}^{z} 》,\right.\right.
$$

with

$$
\begin{aligned}
& F_{q}(t)=\pi\left[-2 \bar{V}_{q} S_{q}^{x} A_{q}-J_{q}\left(S_{q^{z}}^{z} S_{q^{\prime}}^{x}+S_{q^{\prime}}^{x} S_{q^{\prime}}^{z}\right)-\frac{1}{2}\right. \\
& \cdot J_{q}^{\prime}\left(S_{q}^{x} S_{q^{\prime}}^{z} S_{-q}^{z} S_{-q^{\prime}}^{z}+S_{q^{\prime}}^{z} S_{q^{x}}^{x} S_{-q^{z}}^{z} S_{-q^{\prime}}^{z}+S_{-q^{2}}^{z} S_{q^{z}}^{z} S_{q^{\prime}}^{x} S_{-q^{\prime}}^{z}\right. \\
& \left.\left.+S_{-q^{\prime}}^{z} S_{q}^{z} S_{q^{\prime}}^{z} S_{-q}^{x}\right)\right] .
\end{aligned}
$$

$E(\omega)$ is calculated by differentiating (5) twice with respect to " $t$ " using Hamiltonian (1) and then taking Fourier transformation; one obtains

$$
E(\omega)=\frac{\pi\left(\omega^{2}-\Omega^{2}\right) G_{q q^{\prime}}^{z z}}{j \Omega}-\frac{\bar{E}(\omega)}{j},
$$

and higher order Green's functions

$$
\bar{E}(\omega)=\left\langle F_{q}(t) ; F_{q^{\prime}}\left(t^{\prime}\right) 》\right.
$$


with

$$
\begin{aligned}
& F_{q^{\prime}}\left(t^{\prime}\right)=\pi\left[-2 \bar{V}_{q^{\prime}} S_{q}^{x} A_{q}-J_{q}\left(S_{q^{x}}^{x} S_{q^{\prime}}^{z}+S_{q^{\prime}}^{x} S_{q^{\prime}}^{z}\right)-\frac{1}{2}\right. \\
& \cdot J_{q}^{\prime}\left(S_{q}^{x} S_{q^{\prime}}^{z} S_{-q}^{z} S_{-q^{\prime}}^{z}+S_{q^{\prime}}^{z} S_{q^{x}}^{x} S_{-q^{z}}^{z} S_{-q^{\prime}}^{z}+S_{-q^{z}}^{z} S_{q^{\prime}}^{z} S_{q^{\prime}}^{x} S_{-q^{\prime}}^{z}\right. \\
& \left.\left.\quad+S_{-q^{\prime}}^{z} S_{q}^{z} S_{q^{\prime}}^{z} S_{-q}^{x}\right)\right] .
\end{aligned}
$$

Substituting the value of $\bar{E}(\omega)$ from (7) into (4) and using Dyson's equation, one obtains

$$
G_{q q^{\prime}}^{z z}(\omega+j \varepsilon)=\frac{\Omega\left\langle S_{q}^{x}\right\rangle \delta_{q q^{\prime}}}{\pi\left[\omega^{2}-\widetilde{\Omega}-\left(\Omega / 2 \pi\left\langle S_{q}^{x}\right\rangle\right) \overline{\bar{E}}(\omega)\right]},
$$

where the renormalized frequency is

$$
\widetilde{\Omega}^{2}=a^{2}+b^{2}-b c,
$$

with

$$
\begin{aligned}
& a=J_{0}\left\langle S_{q}^{z}\right\rangle+J_{0}^{\prime}\left\langle S_{q}^{z}\right\rangle^{3}, \\
& b=2 \Omega, \\
& c=J_{0}\left\langle S_{q}^{x}\right\rangle+3 J_{0}^{\prime}\left\langle S_{q}^{x}\right\rangle\left\langle S_{q}^{z}\right\rangle^{2}, \\
& J_{0}=\sum_{q} J_{q}, \\
& J_{0}^{\prime}=\sum_{q^{\prime}} J_{q^{\prime}}^{\prime} .
\end{aligned}
$$

Higher order Green's functions $\overline{\bar{E}}(\omega)$ are calculated using symmetrical decoupling scheme, the cross combinations are not considered because they do not contribute significantly, and (10) can be written as

$$
\operatorname{Limit}_{\varepsilon \rightarrow 0} G_{q q^{\prime}}^{z z}(\omega+j \varepsilon)=\frac{\Omega\left\langle S_{q}^{x}\right\rangle \delta_{q q^{\prime}}}{\pi\left[\omega^{2}-\widetilde{\Omega}^{2}+j \Gamma_{s}(q, \omega)\right]},
$$

where $\widetilde{\Omega}$ is the proton renormalized frequency of the coupled system, which on solving self-consistently takes the form

$$
\widetilde{\Omega}^{2}=\widetilde{\Omega}^{2}+2 \Omega \Delta_{s}(q, \omega) .
$$

The real and imaginary parts of (12) are obtained by using the formula

$$
\operatorname{limit}_{\varepsilon \rightarrow 0} \frac{1}{x \pm j \varepsilon}=\frac{1}{x} \mp j \pi \delta(x) .
$$

$\Delta_{s}(q, \omega)$ and $\Gamma_{s}(q, \omega)$ represent collective proton mode frequency shift and width given as

$$
\begin{aligned}
\Delta_{s}(q, \omega)= & \frac{2 \bar{V}_{q}^{2} \omega_{q}\left\langle S_{q}^{x}\right\rangle\left(\omega^{2}-\widetilde{\omega}_{q}^{2}\right)}{\Omega\left[\left(\omega^{2}-\widetilde{\omega}_{q}^{2}\right)^{2}+4 \omega_{q}^{2} \Gamma_{p}^{2}\right]}+\frac{b c^{2}}{\left(\omega^{2}-\widetilde{\Omega}^{2}\right)} \\
& +\frac{a^{2} \widehat{\Omega}^{2}}{\left(\omega^{2}-\widehat{\Omega}^{2}\right)},
\end{aligned}
$$

with

$$
\begin{aligned}
\widehat{\Omega}^{2}= & \left(a^{2}+n_{q} \bar{V}_{q}^{2}\right), \\
\Gamma_{s}(q, \omega)= & \frac{-4 \pi \bar{V}_{q}^{2} \omega_{q}^{2}\left\langle S_{q}^{x}\right\rangle \delta_{q q}^{\prime} \Gamma_{p}}{\Omega\left[\left(\omega^{2}-\widetilde{\tilde{\omega}}_{q}^{2}\right)^{2}+4 \omega_{q}^{2} \Gamma_{p}^{2}\right]} \\
& +\frac{\pi b c^{2}}{2 \widetilde{\Omega}}\{\delta(\omega-\widetilde{\Omega})-\delta(\omega+\widetilde{\Omega})\} \\
& +\frac{\pi a^{2} \widehat{\Omega}}{2 b}\{\delta(\omega-\widehat{\Omega})-\delta(\omega+\widehat{\Omega})\} .
\end{aligned}
$$

2.2. Collective Phonon Half Width and Mode Frequency Shift. The acoustic phonon frequency width and shift are obtained analogously from acoustic phonon Green's function:

$$
G\left(t-t^{\prime}\right)=\left\langle\left\langle A_{q} ; A_{q^{\prime}}^{+}\right\rangle\right.
$$

Differentiating (18) twice with respect to time " $t$," using Hamiltonian (1) taking Fourier transformation, one has

$$
G_{p}(q, \omega)=\frac{\omega_{q} \delta_{q q^{\prime}}}{\pi\left[\left(\omega^{2}-\widetilde{\tilde{\omega}}_{q}^{2}\right)+2 j \omega_{q} \Gamma_{p}(q, \omega)\right]},
$$

where

$$
\begin{aligned}
& \widetilde{\tilde{\omega}}_{q}^{2}=\widetilde{\omega}_{q}^{2}+2 \omega_{q} \Delta_{p}(q, \omega)=\widetilde{\omega}_{q}^{2}+2 \omega_{q} \sum_{i=1}^{3} G_{i p}^{\prime}(q, \omega), \\
& \widetilde{\omega}_{q}^{2}=\omega_{q}^{2}+8 \omega_{q}\left(2 V_{3}+V_{4}\right) \operatorname{coth}\left(\frac{\beta \omega_{q^{\prime}}}{2}\right) .
\end{aligned}
$$

Higher order Green's functions are evaluated without any decoupling and using renormalized Hamiltonian. Putting the evaluated value of higher order Green's function in (19), one gets

$$
G_{p}(\omega)=\frac{\omega_{q} \delta_{q q^{\prime}}}{\pi\left[\omega^{2}-\widetilde{\tilde{\omega}}_{q}^{2}-P(q, \omega)\right]} .
$$

The real part of $P(q, \omega)$ is obtained by using (14) and the collective mode frequency shift is obtained as

$$
\Delta_{p}(q, \omega)=\operatorname{Re} P(q, \omega)=\sum_{i=1}^{3} G_{i p}^{\prime}(q, \omega),
$$


with

$$
\begin{aligned}
& G_{1 p}^{\prime}(q, \omega)=\frac{2 \bar{V}_{q^{2}}^{2}\left\langle\left\langle S_{q}^{x}\right\rangle\left(\omega^{2}-\widetilde{\widetilde{\Omega}}^{2}\right) \delta_{q q^{\prime}}\right.}{\omega_{q}\left[\left(\omega^{2}-\widetilde{\Omega}^{2}\right)^{2}+4 \Omega^{2} \Gamma_{s}^{2}(q, \omega)\right]}, \\
& G_{2 p}^{\prime}(q, \omega)=\frac{24 \sum_{q} V_{3}^{2}\left(q q^{\prime}\right) \omega_{q} n_{q}}{\widetilde{\omega}\left(\omega^{2}-4 \widetilde{\omega}_{q}^{2}\right)}, \\
& G_{3 p}^{\prime}(q, \omega) \\
& =\frac{24 \sum_{q} V_{4}^{2}\left(q q^{\prime}\right) \omega_{q} \omega_{q^{\prime}}}{\widetilde{\omega}_{q}^{2} \widetilde{\omega}_{q^{\prime}}}\left[\frac{\left(1+2 n_{q} n_{q^{\prime}}+n_{q}^{2}\right)\left(2 \widetilde{\omega}_{q}+\widetilde{\omega}_{q^{\prime}}\right)}{\omega^{2}-\left(2 \widetilde{\omega}_{q}+\widetilde{\omega}_{q^{\prime}}\right)^{2}}\right. \\
& \left.+\frac{\left(n_{q}^{2}-1\right)\left(2 \widetilde{\omega}_{q}+\widetilde{\omega}_{q^{\prime}}\right)}{\omega^{2}-\left(2 \widetilde{\omega}_{q}-\widetilde{\omega}_{q^{\prime}}\right)^{2}}+\frac{2 \widetilde{\omega}_{q^{\prime}}\left(n_{q}^{2}-1\right)}{\left(\omega^{2}-\omega_{q^{\prime}}^{2}\right)}\right] \\
& +\frac{72 \sum_{q} V_{4}^{2}\left(q q^{\prime}\right) \omega_{q}^{2}}{\widetilde{\omega}_{q}^{2}}\left[\frac{\left(1+3 n_{q}^{2}\right)}{\left(\omega^{2}-9 \widetilde{\omega}_{q}^{2}\right)}+\frac{\left(n_{q}^{2}-1\right)}{\left(\omega^{2}-\omega_{q^{\prime}}^{2}\right)}\right],
\end{aligned}
$$

where $n_{q}=\left(\omega_{q} / \widetilde{\omega}_{q}\right) \operatorname{coth}\left(\beta \widetilde{\omega}_{q} / 2\right)$ is occupation number and $\beta=\left(k_{B} T\right)^{-1}, k_{B}$ being Boltzmann's constant and $T$ being the absolute temperature. Calculating (20) self-consistently and approximating, the collective mode frequency is given by

$$
\begin{aligned}
\widetilde{\widetilde{\omega}}_{q \pm}^{2}= & \frac{1}{2}\left(\widetilde{\omega}_{q}^{2}+\widetilde{\widetilde{\Omega}}^{2}\right) \\
& \pm \frac{1}{2}\left[\left(\widetilde{\omega}_{q}^{2}-\widetilde{\widetilde{\Omega}}^{2}\right)^{2}+16 \bar{V}_{q}^{2} \omega_{q} \Omega\left\langle S^{x}\right\rangle\right]^{1 / 2} .
\end{aligned}
$$

Equation (22a) is obtained using model Hamiltonian (1), and (22b) and (22c) are obtained without decoupling and using the renormalized Hamiltonian

$$
\begin{aligned}
H_{\text {ren }}= & -2 \Omega \sum_{q} S_{q}^{x}-\frac{1}{2} \sum_{q q^{\prime}} J_{q} S_{q}^{z} S_{q^{\prime}}^{z} \\
& -\frac{1}{4} \sum_{q q^{\prime}-q-q^{\prime}} J_{q}^{\prime} S_{q^{z}}^{z} S_{q^{\prime}}^{z} S_{-q}^{z} S_{-q^{\prime}}^{z} \\
& +\frac{1}{4} \sum_{q} \frac{\omega_{q}^{2}}{\omega_{q}}\left(A_{k}^{+} A_{k}+B_{k}^{+} B_{k}\right) .
\end{aligned}
$$

The imaginary part of $P(q, \omega)$ is obtained by using (19) and collective phonon half width is obtained as

$$
\Gamma_{p}(q, \omega)=\pi \operatorname{Im} P(q, \omega)=\pi \sum_{i=3}^{3} G_{i p}^{\prime \prime}(q, \omega) .
$$

2.3. Order Parameter Values of $\left\langle S_{q}^{x}\right\rangle,\left\langle S_{q}^{y}\right\rangle$, and $\left\langle S_{q}^{z}\right\rangle$. The expectation values of the proton collective mode component at site " $q$ " have been obtained by Blinc and Zeks [26] as

$$
\begin{aligned}
& \left\langle S_{q}^{x}\right\rangle=\frac{\Omega}{\widetilde{\Omega}} \tanh \left(\frac{\beta \widetilde{\Omega}}{2}\right), \\
& \left\langle S_{q}^{y}\right\rangle=0, \\
& \left\langle S_{q}^{z}\right\rangle=\frac{a}{2 \widetilde{\Omega}} \tanh \left(\frac{\beta \widetilde{\Omega}}{2}\right) .
\end{aligned}
$$

In PE phase $\left(T>T_{c}\right),(26)$ represent a system of $3 N$ equations for the average value of the collective mode components. The solution of this system will, however, be stable only if they minimize the free energy, that is, if $\left\langle S_{q}^{z}\right\rangle=\left\langle S_{q}^{y}\right\rangle=0$, and so

$$
\begin{aligned}
\left\langle S_{q}^{x}\right\rangle= & \frac{\Omega}{\left(4 \Omega^{2}-2 \Omega J_{0}\left\langle S_{q}^{x}\right\rangle\right)^{1 / 2}} \\
& \cdot \tanh \left(\frac{\left(4 \Omega^{2}-2 \Omega J_{0}\left\langle S_{q}^{x}\right\rangle\right)^{1 / 2}}{2 k_{B} T}\right),
\end{aligned}
$$

in the ferroelectric phase $\left(T<T_{c}\right)$ [27]. Consider

$$
\begin{aligned}
& \left\langle S_{q}^{x}\right\rangle=\frac{2 \Omega}{J_{0}+J_{0}^{\prime}\left\langle S_{q}^{z}\right\rangle^{2}}, \\
& \left\langle S_{q}^{y}\right\rangle=0, \\
& \left\langle S_{q}^{z}\right\rangle=\frac{1}{2} \tanh \left(\frac{a}{2 k_{B} T}\right) .
\end{aligned}
$$

2.4. Dielectric Constant and Tangent Loss. Following Kubo [28] and Zubarev [25] the real part of dielectric constant is given by

$$
\varepsilon^{\prime}(\omega)-1=-8 \pi^{2} N \mu^{2} G^{\prime}(\omega),
$$

where $\mu$ is the effective dipole moment per unit cell and $N$ is the number of unit cells in the sample.

The dielectric loss $(\tan \delta)$ is defined as the ratio of imaginary and real parts of dielectric constant and can be written as

$$
\tan \delta=\frac{G^{\prime \prime}(\omega)}{G^{\prime}(\omega)}
$$

Thus retarded phonon Green's function is enough to determine the dielectric constant and loss tangent. Using (29) and (22), the real part of dielectric constant can be written as

$$
\varepsilon^{\prime}(\omega)-1=\frac{-8 \pi N \mu^{2} \widetilde{\omega}\left(\omega^{2}-\widetilde{\tilde{\omega}}^{2}\right)}{\left[\left(\omega^{2}-\widetilde{\tilde{\omega}}^{2}\right)^{2}+4 \omega^{2} \Gamma_{p}^{2}(\omega)\right]} .
$$


For the experimental range of frequencies $\omega \ll \widetilde{\widetilde{\omega}}$ (as well as $\omega \tau_{p} \ll 1$ for KDP crystals), (31) can be reduced to

$$
\varepsilon^{\prime}(\omega) \cong \frac{8 \pi N \mu^{2} \widetilde{\omega}}{\widetilde{\tilde{\omega}}^{2}}, \quad \text { as } \varepsilon^{\prime}(\omega) \gg 1,
$$

where $\widetilde{\widetilde{\omega}}^{2}$ is given by (20) and $\widetilde{\omega}$ is given by (20a).

The tangent loss is given by

$$
\tan \delta=\frac{\varepsilon^{\prime \prime}}{\varepsilon}=\frac{-\omega \Gamma_{p}}{\left[\left(\omega^{2}-\widetilde{\tilde{\omega}}^{2}\right)\right]},
$$

where $\Gamma_{p}$ is given by (25). For $\omega \ll \widetilde{\widetilde{\omega}}$, (33) becomes

$$
\tan \delta=\frac{-\omega \Gamma_{p}}{\widetilde{\widetilde{\omega}}^{2}}=\frac{\omega\left(\alpha+\beta T+\gamma T^{2}\right)}{\left(T-T_{c}\right)},
$$

where $\alpha$ is harmonic and defect contribution, $\beta$ and $\gamma$ are due to three and four phonon anharmonic interaction terms of the lattice.

2.5. Ultrasonic Attenuation. The expression for ultrasonic attenuation constant is given by

$$
\alpha_{p}(q, \omega)=\frac{\Gamma_{s}(q, \omega)}{C_{\mu}(q)},
$$

where damping constant is given by $(17)$ and $C_{\mu}(q)$ is the ultrasonic velocity. For small $q$ limit, we obtain the collective proton mode frequency width, for $\omega \ll \tilde{\widetilde{\omega}}$, from (17) expressed as

$$
\begin{aligned}
\Gamma_{s}= & \frac{2 \bar{V}_{q}^{2}\left\langle S^{x}\right\rangle \omega^{2} \tau_{p}}{\widetilde{\widetilde{\omega}}_{q}^{2} \Omega\left(1+\omega^{2} \tau_{p}^{2}\right)} \\
& +\frac{\pi b c^{2}}{2 \widetilde{\Omega}}\{\delta(\omega-\widetilde{\Omega})-\delta(\omega+\widetilde{\Omega})\} \\
& +\frac{\pi a^{2} \widehat{\Omega}}{2 b}\{\delta(\omega-\widehat{\Omega})-\delta(\omega+\widehat{\Omega})\},
\end{aligned}
$$

where $\tau_{p}$ is polarization relaxation time and is given by Litov and Garland [29] as

$$
\tau_{p}=\frac{2 \Gamma_{p}}{\widetilde{\widetilde{\omega}}_{-}^{2}} .
$$

Similar expressions for frequency shift and ultrasonic attenuation have been derived by Zurek [30], using the LandauKhalatnikov theory. If the collective mode damping is neglected, a discontinuity in sound velocity is obtained at $T=T_{c}$, since $\widetilde{\tilde{\omega}}_{-}^{2}$ is proportional to $\left(T_{c}-T\right)$ for $T \leq T_{c}$. the effect of factor $\left(1+\omega^{2} \tau_{p}^{2}\right)^{-1}$ is to smooth out the discontinuity.

The ultrasonic attenuation peaks for $\omega \tau_{p} \cong 1$ and $\omega_{q}$ (i.e., $\widetilde{\widetilde{\omega}}_{-}$) goes to zero as $T \rightarrow T_{c}$. For temperature such as $\omega \tau_{p} \ll 1$, the attenuation will have the form

$$
\alpha(q)=\frac{\Lambda \omega^{2} \tau_{p}}{\left|T_{c}-T\right|}+\alpha_{0},
$$

where

$$
\begin{aligned}
\alpha_{0}= & \frac{\pi b c^{2}}{2 \widetilde{\Omega} C_{\mu}}[\delta(\omega-\widetilde{\Omega})-\delta(\omega+\widetilde{\Omega})] \\
& +\frac{\pi a^{2} \widehat{\Omega}}{2 b C_{\mu}}[\delta(\omega-\widehat{\Omega})-\delta(\omega+\widehat{\Omega})], \\
\Lambda= & \frac{2 \bar{V}_{q}^{2}\left\langle S^{x}\right\rangle}{\Omega C_{\mu} \gamma} .
\end{aligned}
$$

The dependence of ultrasonic attenuation on applied frequency $(\omega)$ and the collective mode frequency $\left(\tilde{\widetilde{\omega}}_{-}\right)$may be expected to apply also to the displacive phase transition [31], for which the coupling is linear to the strain and bilinear to the soft optical normal mode coordinates, independent of whether the phonon instability occurs at $q=0, q=q_{k}$, or at a general point in the Brillouin zone. Though the linear dependence of $\left(\widetilde{\tilde{\omega}}_{-}\right)$on $\left(T-T_{c}\right)$ has been assumed for $T \cong T_{c}$, this dependence presumably breaks down sufficiently close to $T_{c}$.

2.6. Transition Temperature. In the PE phase $\left\langle S_{q}^{z}\right\rangle=0$, and the stability limit of PE phase is determine by the temperature where $\widetilde{\widetilde{\omega}}_{q_{-}}$approaches zero as $T \rightarrow T_{c}$. Consider

$$
\begin{aligned}
& \widetilde{\widetilde{\omega}}_{q^{-}}^{2}=\left(\frac{\partial \widetilde{\tilde{\omega}}_{q^{-}}^{2}}{\partial T}\right)_{T=T_{c}}\left(T-T_{c}\right), \\
& \widetilde{\widetilde{\omega}}_{q^{-}}^{2} \cong \gamma\left(T-T_{c}\right),
\end{aligned}
$$

using (13), where

$$
\gamma=\frac{\Omega^{2} \widehat{J}}{\left[k_{B} T_{c}^{2} \cosh ^{2}\left(\Omega / k_{B} T_{c}\right)\right]},
$$

with effective exchange coupling constant

$$
\widehat{J}=J_{0}+\left.\frac{2 \bar{V}_{q}^{2} \omega_{q}}{\widetilde{\omega}_{q}}\right|_{T=T_{c}}
$$

as well as transition temperature

$$
T_{c}=\Omega\left[k_{\beta} \tanh ^{-1}\left(\frac{4 \Omega}{\widehat{J}}\right)\right]^{-1} .
$$

\section{Comparison with Experiments and Discussion}

3.1. Numerical Calculations. The parameters in our calculation are listed in Table 1. The calculated values of $\left\langle S_{q}^{z}\right\rangle$ and $\left\langle S_{q}^{x}\right\rangle$ for KDP-type crystals for different values of four-body coupling coefficient $\left(J^{\prime}\right)$, collective phonon mode frequency 
TABLE 1: Blinc-de Gennes model parameters for KDP-type crystals as given by Ganguli et al. [23].

\begin{tabular}{lccccccc}
\hline$\Omega\left(\mathrm{cm}^{-1}\right)$ & $J\left(\mathrm{~cm}^{-1}\right)$ & $J^{\prime}\left(\mathrm{cm}^{-1}\right)$ & $V_{i k}\left(\mathrm{~cm}^{-1}\right)$ & $\omega_{k}\left(\mathrm{~cm}^{-1}\right)$ & $\mu \times 10^{18}(\mathrm{cgs})$ & $T_{c}(\mathrm{~K})$ & $V / k T_{c}$ \\
\hline 82 & 334 & 440 & 25.56 & 153 & 1.8 & 123 & 0.299 \\
\hline
\end{tabular}

TABLE 2: Calculated values of $\left\langle S_{q}^{z}\right\rangle$ and $\left\langle S_{q}^{x}\right\rangle$ for KDP crystal for different values of four-body coupling coefficient $\left(J^{\prime}\right)$.

\begin{tabular}{|c|c|c|c|c|c|c|}
\hline \multirow{3}{*}{ Temperature (K) } & \multicolumn{3}{|c|}{$\left\langle S_{q}^{z}\right\rangle$} & \multicolumn{3}{|c|}{$\left\langle S_{q}^{x}\right\rangle$} \\
\hline & $J^{\prime}=J / 3$ & $J^{\prime}=4 J / 3$ & $J^{\prime}=7 J / 3$ & $J^{\prime}=J / 3$ & $J^{\prime}=4 J / 3$ & $J^{\prime}=7 J / 3$ \\
\hline & & & Present study & & & Present study \\
\hline 20 & 0.500 & 0.499 & 0.499 & 0.454 & 0.369 & 0.311 \\
\hline 40 & 0.498 & 0.499 & 0.499 & 0.455 & 0.369 & 0.311 \\
\hline 60 & 0.459 & 0.481 & 0.498 & 0.460 & 0.376 & 0.312 \\
\hline 80 & 0.257 & 0.278 & 0.487 & 0.482 & 0.446 & 0.317 \\
\hline 100 & 0.137 & 0.143 & 0.454 & 0.489 & 0.479 & 0.332 \\
\hline 120 & 0.102 & 0.104 & 0.409 & 0.490 & 0.485 & 0.354 \\
\hline 122 & 0.100 & 0.100 & 0.403 & 0.491 & 0.486 & 0.357 \\
\hline 123 & 0.000 & 0.000 & 0.400 & 0.492 & 0.492 & 0.358 \\
\hline 125 & 0.000 & 0.000 & 0.398 & 0.484 & 0.484 & 0.449 \\
\hline 130 & 0.000 & 0.000 & 0.389 & 0.468 & 0.468 & 0.457 \\
\hline 135 & 0.000 & 0.000 & 0.373 & 0.446 & 0.446 & 0.443 \\
\hline 140 & 0.000 & 0.000 & 0.352 & 0.426 & 0.426 & 0.436 \\
\hline 145 & 0.000 & 0.000 & 0.320 & 0.408 & 0.408 & 0.410 \\
\hline 150 & 0.000 & 0.000 & 0.300 & 0.396 & 0.396 & 0.392 \\
\hline
\end{tabular}

TABLE 3: Calculated values of $\widetilde{\widetilde{\omega}}_{-}, \varepsilon_{a}(0), \varepsilon_{c}(0), \tan \delta_{a}$, and $\tan \delta_{c}$ for KDP-type crystals.

\begin{tabular}{|c|c|c|c|c|c|}
\hline $\begin{array}{l}\text { Temperature } \\
(\mathrm{K})\end{array}$ & $\begin{array}{c}\text { Collective } \\
\text { phonon mode } \\
\text { frequency } \\
\widetilde{\widetilde{\omega}}_{-}\left(\mathrm{cm}^{-1}\right) \\
\text { (present study) }\end{array}$ & $\begin{array}{c}\text { Transverse } \\
\text { dielectric } \\
\text { constant } \\
\varepsilon_{a}(0) \\
\text { (present study) }\end{array}$ & $\begin{array}{c}\text { Observed } \\
\text { dielectric } \\
\text { constant } \\
\varepsilon_{c}(0) \\
\text { (present study) }\end{array}$ & $\begin{array}{l}\text { Loss tangent } \\
\text { along the } \\
a \text {-axis } \\
\left(\tan \delta_{a}\right) \\
\text { (present study) }\end{array}$ & $\begin{array}{l}\text { Loss tangent } \\
\text { along the } \\
c \text {-axis } \\
\left(\tan \delta_{c}\right) \\
\text { (present study) }\end{array}$ \\
\hline 125 & 45.65 & 63 & 35741 & 0.004 & 0.067 \\
\hline 130 & 57.04 & 62 & 6144 & 0.00398 & 0.035 \\
\hline 135 & 58.69 & 61 & 2286 & 0.00397 & 0.0261 \\
\hline 140 & 63.04 & 60 & 874 & 0.00396 & 0.0255 \\
\hline 145 & 65.91 & 59 & 486 & 0.00365 & 0.0250 \\
\hline
\end{tabular}

$\left(\widetilde{\widetilde{\omega}}_{-}\right)$, transverse dielectric constant $\left\{\varepsilon_{a}(o)\right\}$, observed dielectric constant $\left\{\varepsilon_{c}(o)\right\}$, and tangent loss along $a$-axis $\left(\tan \delta_{a}\right)$ and $c$-axis $\left(\tan \delta_{c}\right.$ ) for KDP-type crystals are listed in Tables 2 and 3. Their variations with temperature are shown in Figures $1-4$.

3.2. Temperature Dependence of $\left\langle S_{q}^{z}\right\rangle$ and $\left\langle S_{q}^{x}\right\rangle$ for KDP-Type Crystal for Different Values of $J^{\prime}$. Using Blinc-de Gennes model parameter values for KDP-type ferroelectrics crystals as given by Ganguli et al. [23], putting these values into (27) and (28), we have calculated temperature dependence of $\left\langle S_{q}^{z}\right\rangle$ and $\left\langle S_{q}^{x}\right\rangle$ for KDP-type crystal for different values of $J^{\prime}$, and variation is shown in Figure 1.

In Figure 1, the curve (a) is the case of $J^{\prime}=J / 3<4 J / 3$, curve (b) is $J^{\prime}=4 J / 3<7 J / 3$, and curve (c) is $J^{\prime}=7 J / 3>$ $4 J / 3$. In curves (a) and (b) the value of $\left\langle S_{q}^{z}\right\rangle$ increases to the saturated value 0.5 from zero, when temperature decreases from transition temperature. That is the case of second-order phase transition. But in curve (c) the change of $\left\langle S_{q}^{z}\right\rangle$ with the temperature starts from a nonzero value $\left\langle S_{q}^{z}\right\rangle$ at point "A"; that is to say, when temperature decreases $\left\langle S_{q}^{z}\right\rangle$ increases to the saturation value from the finite value of $\left\langle S_{q}^{z}\right\rangle$. This is the case of first-order phase transition. The temperature at point " $A$ " is transition temperature, and the value of $\left\langle S_{q}^{z}\right\rangle$ at " $A$ " is the discontinuity of $\left\langle S_{q}^{z}\right\rangle$. The value of $\left\langle S_{q}^{x}\right\rangle$ decreases when temperature decreases in the ferroelectric phase. On the other hand, in PE phase the value of $\left\langle S_{q}^{x}\right\rangle$ decreases when temperature increases from transition temperature.

3.3. Temperature Dependence of Collective Phonon Mode Frequency $\left(\widetilde{\tilde{\omega}}_{-}\right)$in PE Phase. Using (23) and (41) and model 


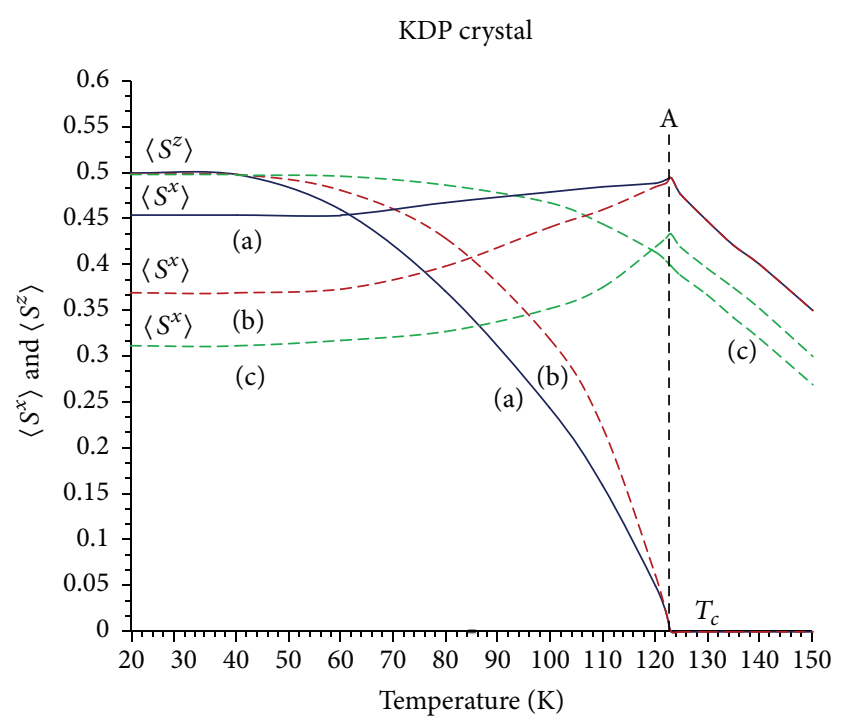

FIGURE 1: Temperature dependence of $\left\langle S_{q}^{z}\right\rangle$ and $\left\langle S_{q}^{x}\right\rangle$ for KDP-type crystal for different values of $J^{\prime}$. (a) $J^{\prime}=J / 3$, (b) $J^{\prime}=4 J / 3$, and (c) $J^{\prime}=7 \mathrm{~J} / 3$ (present study).

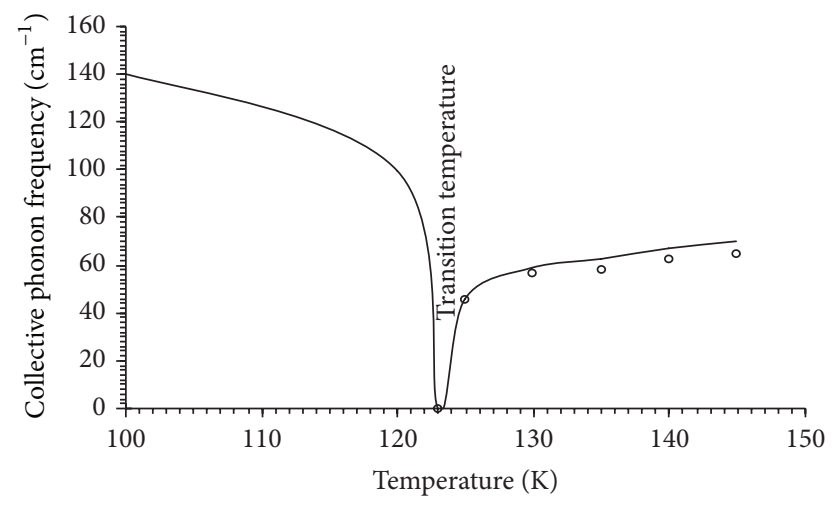

FIgURE 2: Temperature dependence of collective phonon mode frequency $\left(\widetilde{\widetilde{\omega}}_{-}\right)$in PE phase for KDP-type crystals. Present calculation (shown by $\circ$ ) and solid line representing experimental results of Baumgartner [32] and Choi and Lockwood [33].

parameters values from Table 1, the temperature variation of collective phonon mode frequency in PE phase is shown in Figure 2. In the PE phase the temperature dependence of normalized collective phonon frequency enables one to calculate the transition temperature (44), as well as effective exchange coupling constant (43), which increases due to proton-phonon coupling and decreases due to anharmonic interactions. By fitting model values of physical quantities, temperature dependence of collective phonon mode frequency has been calculated which compares well with experimental results of Baumgartner [32] and Choi and Lockwood [33].

3.4. Temperature Dependence of Dielectric Constant. Putting calculated values for different temperature into (32) we obtain dielectric constant for KDP-type crystals. $\widetilde{\widetilde{\omega}}_{+}$mode

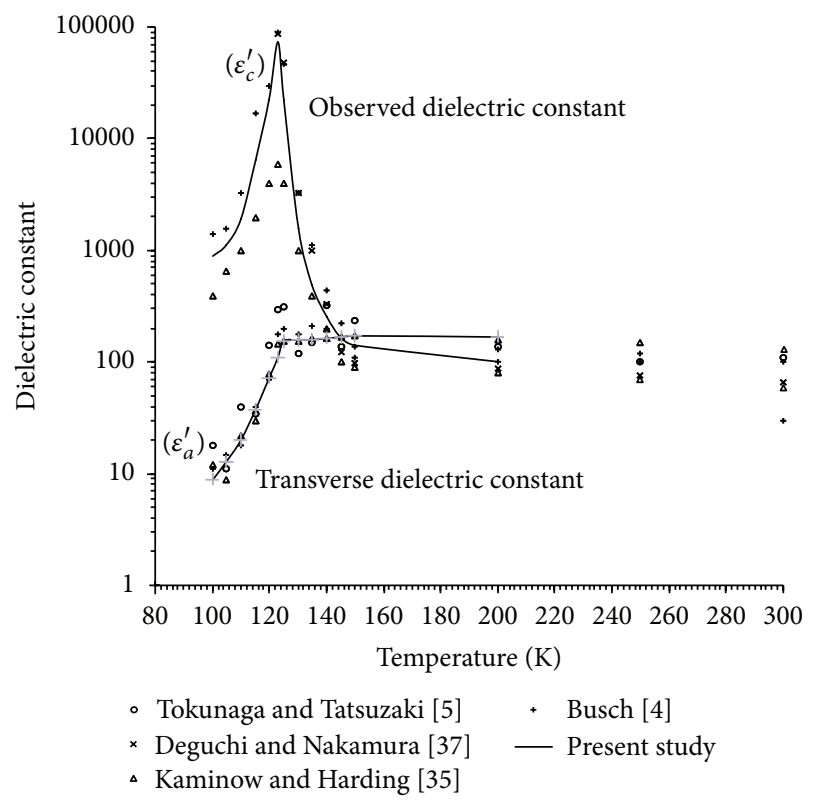

Figure 3: Temperature dependence of dielectric constant for KDP type crystals obtained by Busch [4] (shown by + ), Deguchi and Nakamura [37] (shown by $\times$ ), and Kaminow and Harding [35] (shown by $\triangle$ ), and obtained from Raman intensity [5] (shown by $\circ$ ) and solid line represents the theoretical results of present study.

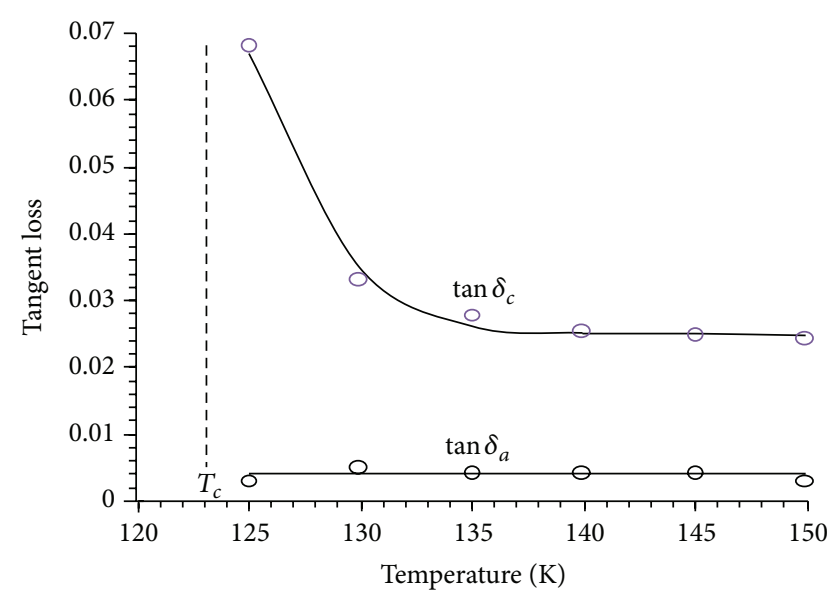

FIgURE 4: Temperature dependence of tangent loss for KDP-type crystals at $9.2 \mathrm{GHz}$ for fields along the $a$-axis $\left(\tan \delta_{a}\right)$, for field along the $c$-axis $\left(\tan \delta_{c}\right)$. Present calculation is represented by 'o', and solid line represents experimental results of Kaminow and Harding [35].

corresponds to transverse $E(x, y)$ mode, which is responsible for the observed transverse dielectric properties of KDP. In the simplest approximation $\widetilde{\widetilde{\omega}}_{+}$can be written $\left(\widetilde{\widetilde{\omega}}_{+}=\right.$ $K_{1}+K_{2} T$ ), where $K_{1}$ and $K_{2}$ are temperature independent parameters. The results for transverse dielectric constant $\varepsilon_{a}^{\prime}$ obtained from the integrated intensity of Raman spectroscopy [34] and those measured by Busch [4] and Kaminow and Harding [35] are shown in Figure 3, together with the theoretical results of Havlin et al. [36]. This indicates that low frequency $\widetilde{\widetilde{\omega}}_{+}\{E(x, y)$ mode $\}$ is closely related to the macroscopic dielectric constant $\varepsilon_{a}^{\prime}$. This also suggests that 
the $E$-mode Raman spectrum originates neither from the second-order Raman scattering nor from the density of states due to the local disorder above $T_{c}$ but from one of the collective modes at the centre of the Brillouin zone. It should be mentioned here that the low frequency $E$ mode continuum appears also in a deuterated KDP (DKDP), although the intensity is about one-third of that of KDP, which indicates the possibility that the spectrum is due to the hydrogen collective motion. Using (23) for $\widetilde{\widetilde{\omega}}_{+}$mode, it can be seen that the $E$-mode collective hydrogen motion has characteristics damping factor which slowly increases as the temperature approaches $T_{c}$, while for that of $\widetilde{\widetilde{\omega}}_{-}\left\{B_{2}(z)\right\}$ soft mode the damping factor slowly decreases down to a finite value, which agrees with the observations of Kaminow and Damen [11].

The present results agree with the behaviour of the observed $E$-mode Raman spectrum in the following aspects: (i) $\widetilde{\tilde{\omega}}_{+}$does not change appreciably as $T \rightarrow T_{c}$ in PE phase, (ii) $\Gamma_{p}\left(\omega_{+}\right)$is weakly dependent on temperature, and (iii) because of the factor $\left(\omega^{2}-\widetilde{\tilde{\omega}}^{2}\right)$ in the numerator of (31), the susceptibility derived changes the corresponding spectrum from a simple overdamped form to a more flat one, like the $E$-mode Raman spectrum of $\mathrm{KH}_{2} \mathrm{PO}_{4}$ [27].

The observed dielectric constant $\left(\varepsilon_{c}^{\prime}\right)$ of $\mathrm{KH}_{2} \mathrm{PO}_{4}$ along $c$-axis is shown in Figure 3. The $\widetilde{\widetilde{\omega}}_{-}\left\{B_{2}(z)\right\}$ mode may be assigned for the observed temperature dependence of $\varepsilon_{c}^{\prime}$. As from (41) $\widetilde{\widetilde{\omega}}_{q}^{2} \propto\left(T-T_{c}\right)$, the real part of the dielectric constant associated with this mode, from (32), can be expressed as

$$
\varepsilon_{c}^{\prime}=\frac{C}{\left(T-T_{c}\right)},
$$

which explains the Curie-Weiss behaviour of dielectric constant along the $c$-axis of $\mathrm{KH}_{2} \mathrm{PO}_{4}$ crystal in the $\mathrm{PE}$ phase observed by Deguchi and Nakamura [37], Busch [4], and Kaminow and Harding [35], shown in Figure 3. For temperature $T \rightarrow T_{c}, \varepsilon_{c}^{\prime}$ tends to maximum value, which is consistent with the theory of Hill and Ichiki [38] for TGS and KDP crystals, while Mason monodisperse theory [39] gives $\varepsilon_{c}^{\prime} \rightarrow 0$ as $T \rightarrow T_{c}$. The origin of this difference in the temperature dependence of $\varepsilon_{c}^{\prime}$ is easily traced back in monodisperse theory, and the critical slowing down of the relaxation time has a dominant effect over the Curie-Weiss law of static dielectric constant, while the Hill-Ichiki theory of distribution function of relaxation time makes contribution to finite $\tau \neq 0$ to $\varepsilon_{c}^{\prime}$ more dominant.

There are actually, however, many cases in which $\varepsilon_{c}^{\prime}$ takes a minimum of finite value at $T=T_{c}$ being neither zero as in Mason's theory nor maximum as in Hill and Ichiki theory.

3.5. Temperature Dependence of Tangent Loss. The tangent loss is associated with damping parameter (25). Damping can be understood as the creation of a virtual polarization mode excited by the transverse electromagnetic radiation and its subsequent decay into the real phonons by scattering from crystal defects, higher order phonon anharmonicities, and so forth. At higher temperature the loss deviates from the
Curie-Weiss type behaviour and increases linearly with temperature. This behaviour suggests that at higher temperatures the phonon anharmonicity contributes significantly to the observed loss.

The calculated values of tangent loss from (33) and (34) and experimental result of Kaminow and Harding [35] are shown in Figure 4 and given in Table 3. The loss was calculated at $9.2 \mathrm{GHz}$ because experimental data is available only at that frequency range. The theoretical results of the present study are in good agreement with experimental results of Kaminow and Harding [35]. The temperature dependence of loss does not appear to be exponential. Thus, third- and fourth-order anharmonicity may be responsible for the observed behaviour of loss tangent. In the microwave frequency rage, an increase in frequency is followed by an increase in transverse and longitudinal dielectric loss tangent. The loss decreases with increase in temperature for KDPtype crystals, in their PE phase. This shows Curie-Weiss type behaviour of the dielectric loss tangent.

In this paper the four-particle cluster model Hamiltonian with the anharmonic contributions up to fourth-order has been taken into consideration in study of dielectric properties and ultrasonic attenuation in KDP-type ferroelectric crystals. Using double time thermal Green's function method and Dyson's equation the collective mode frequencies and widths have been calculated. These parameters lead to the expressions for the dielectric constant and loss tangent. The observed dielectric properties have been explained in terms of present study. The expressions for dielectric constant and loss tangent have been derived and compared with the experimental results. Using Blinc-de Gennes model parameter values given by Ganguli et al. [23] we have calculated temperature variations of these quantities for KDP- type crystals. It is observed that these results are in good agreement with each other and with the results obtained by other methods. The present results reduce to the results of others [22, 23, 40] if the width and shift are neglected. Only Ganguli et al. [23] modified the Ramakrishnan and Tanaka theory by considering anharmonic interaction. Their treatment explains many features of order-disorder ferroelectrics. However, due to insufficient treatment of anharmonic interactions, they could not obtain quantitatively good results and could not describe some very interesting properties, like dielectric properties, acoustic attenuation, and so forth.

Thus, from the present study, it is concluded that the consideration of four-cluster Hamiltonian along with the third- and fourth-order anharmonicities for the KDP-type ferroelectrics leads to the renormalization and stabilization of the relaxational soft mode and the renormalization of the pseudospin exchange interaction constant. The decoupling of the correlations appearing in the dynamical equation after applying Dyson's equation results in shift in frequency and facilitates the calculation of damping parameter, which is related to the loss tangent. The anomalous behaviour in order-disorder KDP-type ferroelectrics finds explanation by the consideration of collective proton-phonon interaction and third- and fourth-order anharmonicities in the fourparticle cluster Hamiltonian. The dielectric properties and ultrasonic attenuation strongly depend on the relaxational 
mode behaviour of stochastic motion of $\mathrm{H}_{2} \mathrm{PO}_{4}$ group in KDP-type ferroelectrics.

\section{Competing Interests}

The authors declare that there is no conflict of interests regarding the publication of this paper.

\section{References}

[1] B. S. Semwal and P. K. Sharma, "Thermal conductivity of an anharmonic crystal," Physical Review B, vol. 5, no. 10, pp. 39093914, 1972.

[2] B. S. Semwal and P. K. Sharma, "Thermal conductivity of an isotopically disordered harmonic crystal," Journal of Mathematical Physics, vol. 15, no. 5, pp. 648-653, 1974.

[3] K. K. Kobayashi, "Dynamical theory of the phase transition in KH2PO4-type ferroelectric crystals," Journal Physics Society Japan, vol. 24, p. 297, 1968.

[4] G. Busch, "Neue seignette-elektrika," Helvetica Physica Acta, vol. 11, no. 4, p. 269, 1938.

[5] M. Tokunaga and I. Tatsuzaki, "Light scattering spectra of polarization fluctuations and models of the phase transition in KDP type ferroelectrics," Phase Transitions, vol. 4, no. 2, pp. 97155, 1984.

[6] S. C. Deorani, U. C. Naithani, and B. S. Semwal, "Fourspin contribution in DKDP," Indian Journal of Pure \& Applied Physics, vol. 37, pp. 215-218, 1999.

[7] T. C. Upadhyay and B. S. Semwal, "Temperature dependence of dielectric tangent loss in KDP and DKDP crystal at microwave frequencies," Indian Journal of Pure \& Applied Physics, vol. 40, pp. 615-619, 2002.

[8] T. C. Upadhyay and B. S. Semwal, "Microwave dielectric tangent losses in KDP and DKDP crystals," Pramana Journal of Physics, vol. 60, no. 3, pp. 525-533, 2003.

[9] T. C. Upadhyay, R. S. Bhandari, and B. S. Semwal, "Dielectric properties of KDP-type ferroelectric crystals in the presence of external electric field," Pramana, vol. 67, no. 3, pp. 547-552, 2006.

[10] H. Yurtseven and S. Şen, "Calculation of the spontaneous polarization and the dielectric constant as a function of temperature for $\mathrm{KH}_{2} \mathrm{PO}_{4}$," Advances in Condensed Matter Physics, vol. 2012, Article ID 259712, 5 pages, 2012.

[11] I. P. Kaminow and T. C. Damen, "Temperature dependence of the Ferroelectric mode in $\mathrm{KH}_{2} \mathrm{PO}_{4}$," Physical Review Letters, vol. 20, no. 20, pp. 1105-1108, 1968.

[12] R. H. Lyddane, R. G. Sachs, and E. Teller, "On the polar vibrations of alkali halides," Physical Review, vol. 59, no. 8, pp. 673-676, 1941.

[13] J. F. Scott, "Soft-mode spectroscopy: experimental studies of structural phase transitions," Reviews of Modern Physics, vol. 46, no. 1, pp. 83-128, 1974.

[14] R. Blinc, "On the isotopic effects in the ferroelectric behaviour of crystals with short hydrogen bonds," Journal of Physics and Chemistry of Solids, vol. 13, no. 3-4, pp. 204-211, 1960.

[15] M. Tokunaga and T. Matsubara, "Theory of ferroelectric phase transition in $\mathrm{KH}_{2} \mathrm{PO}_{4}$ type crystals I," Progress of Theoretical Physics, vol. 35, pp. 581-599, 1996.

[16] P. G. de Gennes, "Collective motions of hydrogen bonds," Solid State Communications, vol. 1, no. 6, pp. 132-137, 1963.
[17] R. S. Katiyar, J. F. Ryan, and J. F. Scott, "Proton-phonon coupling in $\mathrm{CsH}_{2} \mathrm{AsO}_{4}$ and $\mathrm{KH}_{2} \mathrm{AsO}_{4}$," Physical Review B, vol. 4, no. 8, pp. 2635-2638, 1971.

[18] B. Labrenčič, I. Levstek, B. Žekš, R. Blinc, and D. Hadži, "Laser Raman study of quasi-spin wave hydrogen tunneling modes in $\mathrm{KH}_{2} \mathrm{PO}_{4}$ and $\mathrm{KD}_{2} \mathrm{PO}_{4}$," Chemical Physics Letters, vol. 5, no. 7, pp. 441-444, 1970.

[19] K. N. Pak, "Strong coupling between proton and phonon in KDP," Physica Status Solidi B, vol. 60, no. 1, pp. 233-239, 1973.

[20] S. C. Deorani, P. Singh, and M. S. Yadav, "Investigation of deuteration effect on sound attenuation in hydrogen bonded ferroelectric crystals," Material Science Research India, vol. 8, no. 1, pp. 137-141, 2011.

[21] N. S. Panwar and B. S. Semwal, "Soft mode dynamics of ABO3type trigonal ferroelectrics: application to lithium niobate," Indian Journal of Pure \& Applied Physics (IJPAP), vol. 28, p. 706, 1990.

[22] V. Ramakrishnan and T. Tanaka, “Green's-function theory of the ferroelectric phase transition in potassium dihydrogen phosphate (KDP)," Physical Review B, vol. 16, no. 1, pp. 422-426, 1977.

[23] S. Ganguli, D. Nath, and B. K. Chaudhuri, “Green's-function theory of phase transitions in hydrogen-bonded ferroelectric crystals with pseudo-spin-lattice coupled mode model," Physical Review B, vol. 21, no. 7, pp. 2937-2945, 1980.

[24] R. Blinc and B. Žekš, "Proton order-disorder in $\mathrm{KH}_{2} \mathrm{PO}_{4}$-type ferroelectrics: slater theory and ising model in a transverse tunneling field," Ferroelectrics, vol. 72, no. 1, pp. 193-227, 1987.

[25] D. N. Zubarev, "Double-time green functions in statistical physics," Uspekhi Fizicheskikh Nauk, vol. 71, pp. 71-116, 1960.

[26] R. Blinc and B. Zeks, "In soft modes in ferroelectrics and antiferroelectrics," Advance in Physics, vol. 29, p. 701, 1972.

[27] Q. Zikai, Z. Jinbo, and W. Chunlei, "Application of Green's function method to the study of order-disorder ferroelectrics," Ferroelectrics, vol. 101, no. 1, pp. 159-169, 1990.

[28] R. Kubo, "Statistical-mechanical theory of irreversible processes. I. general theory and simple applications to magnetic and conduction problems," Journal of the Physical Society of Japan, vol. 12, no. 6, pp. 570-586, 1957.

[29] E. Litov and C. W. Garland, "Ultrasonic experiments in KDPtype crystals," Ferroelectrics, vol. 72, no. 1, pp. 19-44, 1987.

[30] R. Zurek, Personal Communication, 1969.

[31] E. Pytte, "Soft-mode damping and ultrasonic attenuation at a structural phase transition," Physical Review B, vol. 1, no. 2, pp. 924-930, 1970

[32] H. Baumgartner, "Elektrische sättigungserscheinungen und elektrokalorischer effekt von kaliumphosphat KH2PO4," Helvetica Physica Acta, vol. 24, no. 7, p. 651, 1950.

[33] B. Choi and D. Lockwood, "Dielectric anomalies in $\mathrm{KH}_{2} \mathrm{PO}_{4}$," Ferroelectrics, vol. 106, no. 1, pp. 15-20, 1990.

[34] Y. Takagi and T. Shigenari, "Transverse susceptibility and Emode Raman spectra of a $\mathrm{KH}_{2} \mathrm{PO}_{4}$ crystal," Journal of the Physical Society of Japan, vol. 39, no. 2, pp. 440-447, 1975.

[35] I. P. Kaminow and G. O. Harding, "Complex dielectric constant of $\mathrm{KH}_{2} \mathrm{PO}_{4}$ at $9.2 \mathrm{Gc} / \mathrm{sec}$," Physical Review, vol. 129, no. 4, pp. $1562-1566,1963$.

[36] S. Havlin, E. Litov, and E. A. Uehling, "Transverse susceptibility in $\mathrm{KH}_{2} \mathrm{PO}_{4}$-type crystals," Physical Review B, vol. 9, no. 3, article $1024,1974$. 
[37] K. Deguchi and E. Nakamura, "Deviation from the Curie-Weiss Law in $\mathrm{KH}_{2} \mathrm{PO}_{4}$," Journal of the Physical Society of Japan, vol. 49 , no. 5, pp. 1887-1891, 1980.

[38] R. M. Hill and S. K. Ichiki, "High-frequency behavior of hydrogen-bonded ferroelectrics: triglycine sulphate and $\mathrm{KD}_{2} \mathrm{PO}_{4}$," Physical Review, vol. 132, no. 4, pp. 1603-1608, 1963.

[39] W. P. Mason, "Theory of the ferroelectric effect and clamped dielectric constant of Rochelle salt," Physical Review, vol. 72, no. 9, pp. 854-865, 1947.

[40] H. Konwent, "On the theory of hydrogen-bonded ferroelectrics," Physica Status Solidi B, vol. 28, no. 1, pp. 39-44, 1968. 

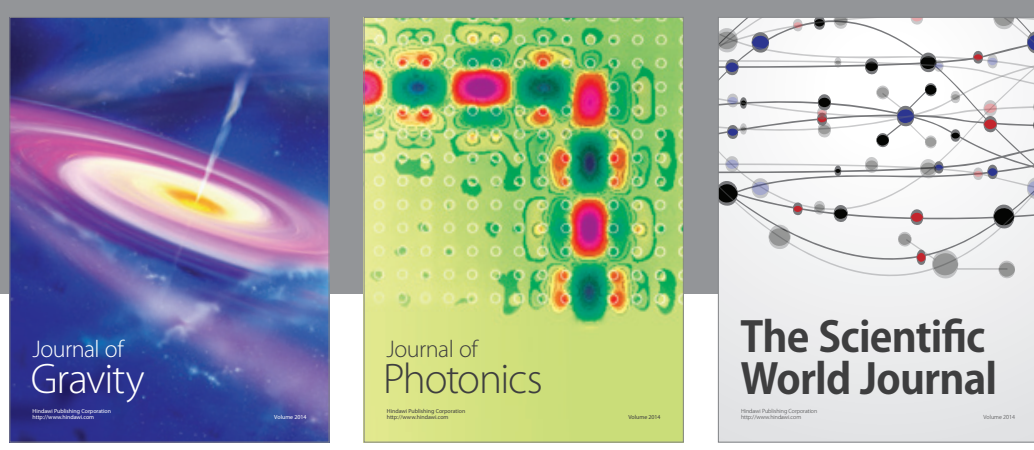

The Scientific World Journal
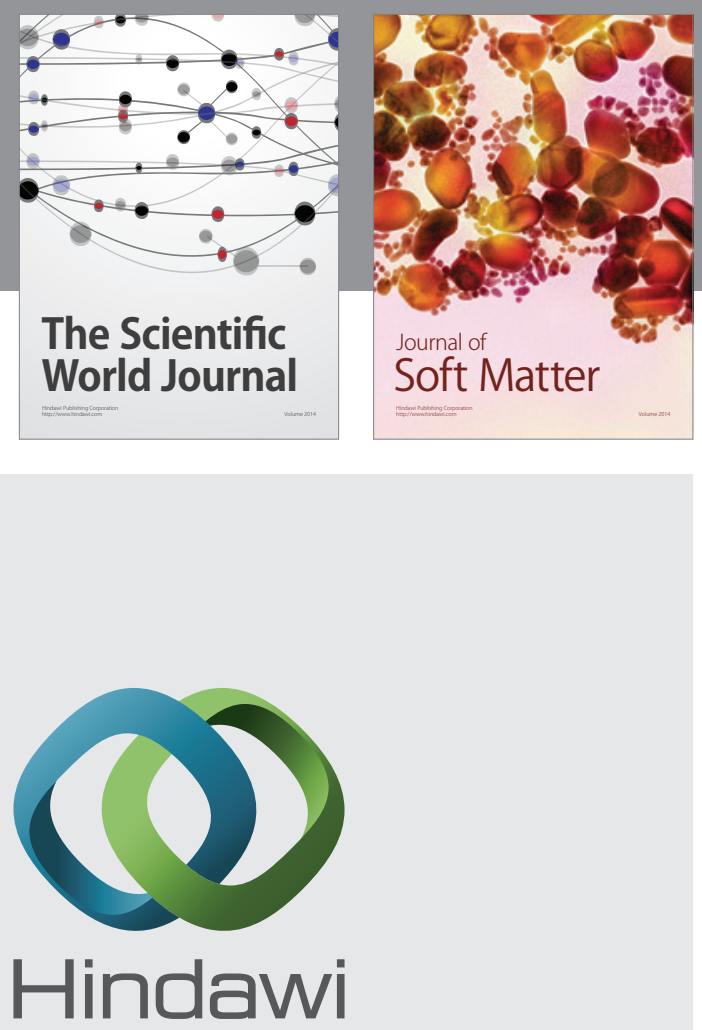

Submit your manuscripts at

http://www.hindawi.com

nternational Journal of

Statistical Mechanics
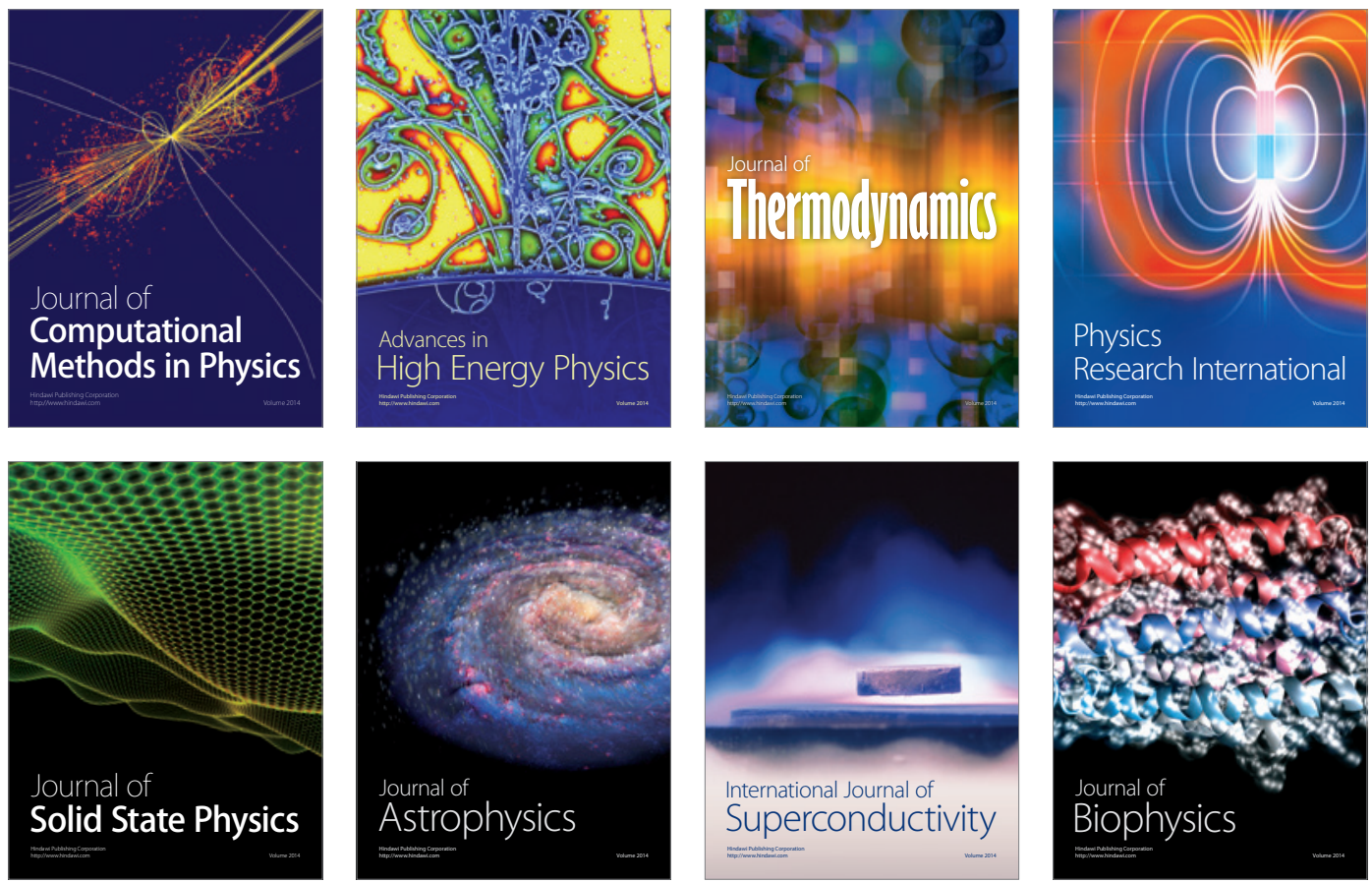
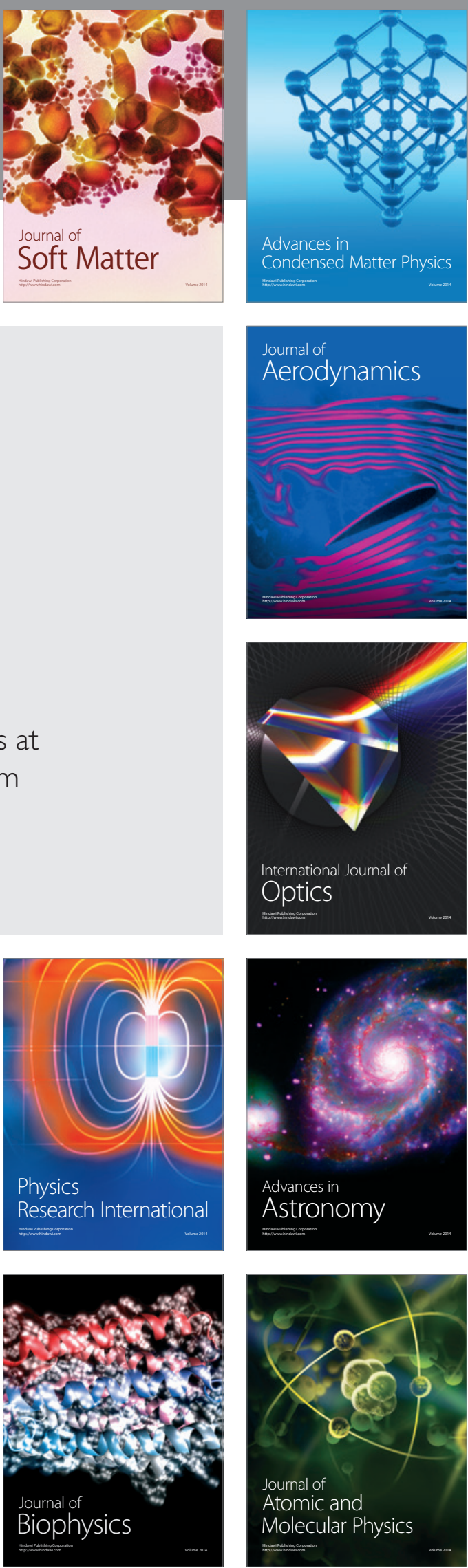